\title{
How to "Detronize" Virus in Crown - Questions to Current Stem Cell Therapies
}

\author{
Mariusz Z. Ratajczak ${ }^{1}$ \\ Published online: 21 April 2020 \\ (C) Springer Science+Business Media, LLC, part of Springer Nature 2020
}

COVID-19, as a pandemic, has took already many precious lives and affects our social habits having tremendous impact on business worldwide with consequences we can not yet foresee. It is also a serious warning that similar viruses may occur in the future and we have to be better prepared for fight at all fronts including at the health care, economy, and social habits level. Unfortunately, no standard treatment for this disease has been discovered to date and several experimental strategies including certain drugs and even cellular therapies has been proposed. It is nothing wrong to try this before vaccine will be developed, so far they do not bring danger of considerable side effects to the patients.

For us as stem cell scientists the most important question is what will be impact of COVID-19 pandemic on stem cell therapies and stem cell research. First, in a very elegant commentary Drs. Broxmeyer and Parker discussed a potential impact of COVID-19 outbreak on procedures related to harvest, processing, and storage of hematopoietic stem cells for transplantation as well other types of stem cells for application in regenerative medicine [1]. It is a risk that this virus as well as other similar viruses that may emerge in the future, will affect the cell therapeutic products, the storage of cryopreserved cells, and their use in clinic. New screening methods and standard protocols have to be developed as has been done in the past with other infectious pathogens. Finally, it is still to early to make any conclusions on late effects of COVID-19 on stem cell compartment in the lung as well as in other tissues.

In this issue of Stem Cell Reviews and Reports we publish two reviews and concept papers. The first one by Dr. Golchin et al. [2] deals with potential application of mesenchymal stroma cells (MSC), as immunomodulatory therapeutics to mitigate cytokine war in the lungs. It is still to early to conclude if this will work and save lives. MSCs have been successfully employed to mitigate graft versus host disease (GvHD) in

Mariusz Z. Ratajczak

mariusz.ratajczak@louisville.edu

1 University of Louisville, Louisville, KY, USA patients undergoing hematopoietic transplants, and several soluble factors and extracellular microvesicles secreted by these cells have evident anti-inflammatory activity. Would they also work effectively in COVID-19 infection? Another paper in this issue of Stem Cell Reviews and Reports from Drs. Pillat and Ulrich discusses some experimental drugs including some anti-malaria compounds such as macrolide-type antibiotic azithriomycin and a drug employed for many years to treat lupus and arthritis that is hydroxychloroquine [3]. The logic behind application of azithriomycin is that COVID-19 uses as entry receptor $\mathrm{CD} 147$ and as postulated interferes with this process, as well as in addition it induces anti-viral responses in lung epithelial cells by elevating interferons and interferon-stimulated proteins to mitigate replication and release of viruses [3]. However, the potential beneficial effect of azithriomycin on COVID-19 has not yet been extensively evaluated. Similarly, more evidence is needed to show effectiveness of hydroxychloroquine being an immunomodulatory drug as well as other antiviral drugs such as remdesivir that have been recently proposed for treatment. Another question is if modulation of Nlrp3 inflammasome that most likely contributes to cytokine war in the lungs could be an effective approach?

At this point physicians and scientists are still trying to develop in addition to social distancing some powerful treatment strategies. If some of the proposed therapies will not work as expected before, we will have in hand effective vaccine or drug, I would like to referee to some a famous quotation from Max Planck "One rule is important in science - only courageous people win". With time we will be able to assess the effectiveness of proposed new experimental therapeutic approaches. This justifies application of MSC or even what is feasible instead of intact MSC, to employ as immunomodulatory agents MSC-derived extracellular microvesicles [4]. Nevertheless, careful and well controlled clinical trials have to be performed that follow an ancient Latin ethical phrase "Primum non nocere" that means "first, do no harm."

I wish all of you "to stay safe from virus" and encourage to explore new therapeutic possibilities with an aim to help the 
suffering world population. We need to "detronize" this virus in crown as soon as possible. This is an important task also for us - stem cell researchers.

\section{References}

1. Golchin A. (2020). Mesenchymal stem cell therapy for COVID-19: present or future Article. Stem Cell Reviews and Reports (this issue).

2. Ulrich, H., Pillat, M.M. (2020) CD147 as a target for COVID-19 treatment: Suggested effects of azithromycin and stem cell engagement (this issue).
3. Broxmeyer, H. E., \& Parker, G. C. (2020). Impact of COVID-19 and future emerging viruses on hematopoietic cell transplantation and other cellular therapies. Stem Cells and Development. https://doi. org/10.1089/scd.2020.0064.

4. Ratajczak, M. Z., Kucia, M., Jadczyk, T., Greco, N. J., Wojakowski, W., Tendera, M., \& Ratajczak, J. (2012 Jun). Pivotal role of paracrine effects in stem cell therapies in regenerative medicine: Can we translate stem cell-secreted paracrine factors and microvesicles into better therapeutic strategies? Leukemia., 26(6), 1166-1173.

Publisher's Note Springer Nature remains neutral with regard to jurisdictional claims in published maps and institutional affiliations. 